\title{
A Study of Myocardial Ischemia Model Induced by Left Coronary Artery Ligation in Rats
}

\author{
Hailin Wang*, Chunyu Cao*, Lianqiang Hui, Ting Liu\#, Yanli Wang, Shuangrong Gao, \\ Yi Zhang, Ran Hao, Chun Li, Chen Zang \\ Institute of Chinese Materia Medica, China Academy of Chinese Medical Sciences, Beijing, China \\ Email: "Itbit@163.com
}

Received 17 March 2016; accepted 24 May 2016; published 27 May 2016

Copyright (C) 2016 by authors and Scientific Research Publishing Inc.

This work is licensed under the Creative Commons Attribution International License (CC BY). http://creativecommons.org/licenses/by/4.0/

(c) (i) Open Access

\begin{abstract}
Objective: Coronary artery was ligated to study the characteristics of myocardial ischemia in rats. Methods: The left anterior descending artery was ligated to establish the rat model of acute myocardial ischemia. All animals were divided into normal control group, sham operation group and model group. 1, 2 and 4 weeks after modeling, ECG (II lead) was recorded, the weight of whole heart and left ventricle were recorded and organ indexes were calculated; myocardial infarct size was determined by TTC; CK, CK-MB, LDH, AST contents of serum were detected; cardiac function was determined by left ventricular intubation via carotid artery and left ventricular was taken to perform pathological observation. Results: 1 week after modeling, compared with the sham operation group, the ECG and heart function index of rats model had significant change, but the myocardial enzymes did not change significantly; 4 weeks after modeling, the ECG and cardiac function of animal models had a recovery trend, but the myocardial enzymes, including CK, CK-MB, LDH, AST, were significantly increased; 1 week after modeling, the left ventricular indexes of model rats were increased; the infarct size was about $30 \%$, myocardial cell necrosis and granulation tissue hyperplasia could be observed in infarction area; with the modeling time extended, from 2 to 4 weeks, the left ventricular and heart indexes of model group were significantly increased; the infarct size was relatively constant, left ventricular became thickly, and fibrous or granulation tissue was significantly proliferated in infarction area under microscope. Conclusion: The indexes of myocardial ischemia induced by coronary artery ligation in rats are different at different time points. The results suggest that the time point should be selected to observe the anti-myocardial ischemia effect of the subjects from different aspects.
\end{abstract}

\footnotetext{
*These authors contributed equally to this work and should be considered co-first authors.

${ }^{\#}$ Corresponding author.
}

How to cite this paper: Wang, H.L., Cao, C.Y., Hui, L.Q., Liu, T., Wang, Y.L., Gao, S.R., Zhang, Y., Hao, R., Li, C. and Zang, C. (2016) A Study of Myocardial Ischemia Model Induced by Left Coronary Artery Ligation in Rats. World Journal of Cardiovascular Diseases, 6, 133-142. http://dx.doi.org/10.4236/wjcd.2016.65014 
Keywords

Coronary Artery Ligation, Myocardial Ischemia, Rat

\section{Introduction}

Acute myocardial infarction (AMI) is a common acute and severe disease in clinic. The incidence of AMI is increasing year by year due to the aging of society, the acceleration of the rhythm of modern life, the change of eating habits and the influence of social and psychological factors. With the change of living habits and the increase of the amount of cholesterol intake, the incidence of AMI is increasing, reaching 45/100,000 to 55/100,000, and it has become one of the major diseases that threaten human health [1].

Left anterior descending artery is the main blood-supply vessels for the left ventricle anterior wall, interventricular septum and left ventricular anterior lateral wall, coronary artery ligation of the left anterior descending coronary artery is the most commonly used method in the study of myocardial infarction. Because of the low cardiac collateral circulation, the high success rate and good reproducibility of model, acute myocardial ischemia induced by ligation of coronary artery in rat has been recognized as a classic model of ischemic cardiomyopathy, which is consistent with the actual onset of myocardial infarction, and is also commonly used in the study of myocardial ischemia pharmacodynamics [2]-[4].

In order to evaluate the effect of drug treatment, it is necessary to observe many aspects, including the changes of electrocardiogram (ECG), myocardial infarct size, cardiac function index, myocardial enzyme spectrum, myocardial pathological changes and so on. However, there are not many related literatures about the change law of the above parameters at different modeling time [5] [6]. If administration time is too short, duo to the effects of some medicine is long-term and slow, such as herbal medicine, it is too short to show the corresponding effect; if the time is too long, because of the strong self-recovery of animals, some of the indicators may show a trend of restoration, it may lead to an incorrect assessment of the efficacy of the drug. For better control of treatment course and more accurate evaluation of the efficacy of drugs, it is important to understand which indicators are more sensitive at different modeling time.

\section{Materials and Methods}

\subsection{Drugs and Reagents}

Creatine Kinase (CK) kit, Creatine Kinase isoenzyme (CK-MB) kit, Lactate Dehydrogenase (LDH) kit and Aspartate Transferase (AST) kit were all purchased from Beijing Wantaiderui Diagnostic Technology Co. Ltd. 2,3,5-triphenyltetrazolium Chloride (TTC) was imported and packed by MYM Biotechnology Company, purity > $98 \%$.

\subsection{Aminals}

Male Sprague-Dawley rats (SPF, 230 - 250 g) were purchased from Beijing Weitonglihua Experimental Animal Technique Co. Ltd [Licence number: SCXK (Jing) 2012-0001]. The animals were kept in a room maintained at a temperature $23^{\circ} \mathrm{C} \pm 3^{\circ} \mathrm{C}$ and a relative humidity of $40 \%-70 \%$ with 12 hours light and 12 hours dark cycle and with approximately 15 times air change per hour. Animals were bred in bottom-meshed stainless steel cages, with filtrated tap water and the fixed-formula rat granula feed. The number of application for welfare and ethic of animal use was 20142005.

\subsection{Instruments}

DH-140B animal respirator was purchased from Zhejiang Medical University medical instruments factory (CHN). Sartorius BSA 8201-CW balance and Sartorius BSA 223S-CW balance were purchased from Sartorius AG (GER). Mp-150 multi-channel physiological recorder was provided by the United States Biopac Company (U.S.A.). Shandon Excelsior ES automatic dehydration machine, Shandon Histocentre 3 paraffin embedded machine, Shandon Finesse 325 slicing machine and Shandon Varistain Gemini full automatic staining machine 
were all purchased from Thermo Shandon Co. Ltd. (UK). Olympus BX51 microscope and image analysis system were purchased from Olympus Company (JPN).

\subsection{Methods}

\subsubsection{Animal Modeling [7]-[9]}

Healthy male SD rats (230 - 250 g) were fixed on board under ether inhalation anesthesia. Pin electrodes were inserted into the skin of the extremities, and the II-lead ECG was recorded as normal ECG. After noninvasive endotracheal intubation by oral, we disinfected the chest of rats, longitudinally cut open a $2 \mathrm{~cm}$ skin incision at the left midclavicular line, bluntly separated the musle layer between the 4th and 5th rib. After opening the chest, we connected animal respirator (37 times/minute, respiratory ratio: 1.25:1, tidal volume: $20 \mathrm{ml}$ ), lightly pressed on the right side of thorax, extruded the heart. Taking the left coronary artery which is between the pulmonary conus and left atrial appendage as a sign, we inserted the needle $2 \mathrm{~mm}$ below the root of the left atrial appendage with $0.5 \mathrm{~mm}$ depth of needle. Then we threaded the surface of myocardium and out near the pulmonary arterial cone by 5/0 suture, rapidly ligated left anterior descending artery, closed the thorax, gently massaged heart to recover the spontaneous breathing of animals, finally sutured the incisions layer by layer. After the operation, each rat was administrated 100,000 U penicillin by intramuscular injection to prevent infection.

\subsubsection{Grouping}

Before operation, animals were randomly divided into three parts according to their weight: normal control part, sham operation part and operation part. The normal control part and the sham operation part were divided into three groups (1, 2 and 4 weeks) respectively, 10 animals in each group.

The left anterior descending artery of the operation part animals were ligated according to the above method. After the operation, that is when the animals recovered spontaneous breathing, the II-lead ECG was immediately recorded as above. Comparison with the preoperative ECG of animals, if the ECG showed significantly raised J points and/or tall and biphasic or inverted T wave and/or Q wave, these were considered as markers of myocardial ischemia, if the above abnormal ECG was not present, it was considered as the ligation position was shifting, the animal modeling was failure and the animal should be abandon. The animals which met the above ECG requirements were randomly divided into three modeling groups (1, 2 and 4 weeks), 11 animals in each group.

The ECG of normal control group without any surgery was recorded under ether anesthesia. In sham operation group, the coronary artery was only threaded by the suture but was not ligated.

At 1, 2 and 4 weeks of modeling, animals in three groups (normal, sham operation and model group) were fasted for 16 hours and intraperitoneally injected $10 \%$ chloral hydrate $\left(3.0 \mathrm{ml} \cdot \mathrm{kg}^{-1}\right)$ to detect the following indicators.

\subsubsection{Detection Index}

1) Detection of the change values of ST segment in ECG [10]

Pin electrodes were inserted into the skin of the extremities, the II-lead ECG were recorded, and the values of ST segment and T wave in ECG were considered as indexes to evaluate the degree of myocardial ischemia.

2) Cardiac function measuring [11]

Left ventricular was intubated. One end of the catheter was connected to pressure transducer which connected to multichannel physiologic recorder. Left ventricular systolic pressure (LVSP), left ventricular end diastolic pressure (LVEDP), the maximum rise rate of left ventricular pressure during isovolumic contraction (+dp/ dtmax), the maximum decline rate of left ventricular pressure during isovolumic diastole ( $-\mathrm{dp} / \mathrm{dtmin})$ and heart rate (HR) were recorded.

3) Detection of serum enzyme

The blood of rat was collected and centrifuged at $4^{\circ} \mathrm{C} 3000 \mathrm{rpm}$ for $15 \mathrm{~min}$. The serum was separated, and the content of CK, LDH, CK-MB and AST were detected by automatic biochemistry analyzer.

4) Organ index

Took the hearts, rinsed with physiological saline, eliminated fat, vessels and other noncardiac tissue, absorbed moisture, weighed the wet weight of hearts, then resected the atrium along coronary sulcus, left the left ventricle, weighed the wet weight of left ventricle, finally according to the body weight of each animal, calculated the whole heart index and left ventricular index (organ index) 


$$
\text { Organ index }(\%)=[\text { organ weight }(\mathrm{g}) / \text { body weight }(\mathrm{g})] \times 100
$$

5) Determination of myocardial infarction size

The left ventricle was taken and weighed. Across sectioned the left ventricular tissue into 1 - $2 \mathrm{~mm}$ from the apex to the base of the heart, rinsed with saline and placed into $1 \%$ TTC solution, incubated for $15 \mathrm{~min}$ at $37^{\circ} \mathrm{C}$, rinsed off the excess dye. The infarction area was not stained, the non-infarcted area was stained red. Carefully cutted uncolored infarction area and weighed its wet weight, calculated the percentage of the weight of ischemic myocardium to left ventricle:

$$
\begin{aligned}
& \text { The range of myocardial infarction } \\
& =\text { the wet weight of the ischemic area/the wet weight of the left ventricle } \times 100 \%
\end{aligned}
$$

6) Histopathological examination

The left ventricle was fixed in $10 \%$ formaldehyde. After alcohol dehydration with, paraffin embedding, slicing and HE staining, the tissue slices were made. Then, they were observed by light microscope. The pathological grading was performed according to the standard in Table 1.

\subsubsection{Statistical Analysis}

Pathological grading score was analysed with non-parametric analysis, comparison among groups was analysed with Kruskal-Wallis test. The rest data, such as organ indexes, biochemical parameters, myocardial infarction size, the values of ST segment and T wave in ECG and other indicators were represented with $\bar{x} \pm$ sd (standard deviation), comparisons among groups were analyzed with one-way analysis of variance (ANOVA); if there was significant difference among groups, the comparison between sham operation group and the normal control group, model group and sham operation group, were analysed with LSD test. Probability values of $P<$ 0.05 were considered significant.

\section{Results}

\subsection{Electrocardiogram}

One week after coronary artery ligation, the ST segment in ECG of model group rat was significantly higher than that in sham operation group $(P<0.01)$, indicating the ligation leaded to myocardial ischemia; at 2 weeks after modeling, the ST segment in ECG of model group rat was still significantly higher than that in sham operation group $(P<0.05)$, but the value of ST segment elevation was lower than that in rats 1 week after modeling; 4 weeks after modeling, the ST segment in ECG of model group rats was still higher than that in sham operation group, but no significant difference was found $(P>0.05)$. Compared with the sham operation group, the elevated value of ST segment in rats after modeling 1,2 and 4 weeks were increased $0.088,0.062$ and $0.067 \mathrm{mv}$ respectively. There

\section{Table 1. The standard of pathological grading.}

\begin{tabular}{ccl}
\hline Pathological score & $\begin{array}{c}\text { Pathological } \\
\text { grading }\end{array}$ & Left ventricular lesions \\
\hline 0 & - & The structure of the heart was normal and no obvious pathological changes were found. \\
\hline & + & $\begin{array}{l}\text { Epicardium was thickened, focal myocardial cells under epicardium were swelling, } \\
\text { degenerative and necrotic, accompanied by granulation tissue or fibrous tissue hyperplasia } \\
\text { and the infiltration of a small amout inflammatory cells. }\end{array}$ \\
3 & $\begin{array}{l}\text { Epicardium was thickened, small patchy myocardial cells under epicardium were necrotic, } \\
\text { granulation tissue or fibrous tissue was mildly hyperplastic, and a small amount of } \\
\text { inflammatory cells were infiltrated. }\end{array}$ \\
& $\begin{array}{l}\text { Epicardium was thickened, patchy myocardial cells under epicardium were necrotic, } \\
\text { accompanied by the hyperplasia of granulation tissue or fibrous tissue and the } \\
\text { infilatration of a small amount inflammatory cell. }\end{array}$ \\
+++ & $\begin{array}{l}\text { Large area myocardium was infarcted (transmural infarct), granulation tissue or fibrous scar } \\
\text { tissue was significantly increased, the ventricular wall was significantly thin. }\end{array}$
\end{tabular}


was no obvious difference in the value of $\mathrm{T}$ wave between the model group and the sham operation group at any modeling time. There were no significant differences in the value of $\mathrm{T}$ wave and ST segment between normal control group and sham operation group after modeling 1, 2, 4 weeks. The results are shown in Figure 1 and Table 2.

Table 2. The value of ST segment and T wave in ECG of myocardial ischemia rats induced by coronary artery ligation at different modeling time ( $\bar{x} \pm \mathrm{sd})$.

\begin{tabular}{ccccc}
\hline Modeling time & Groups & $\mathrm{n}$ & $\mathrm{S}-\mathrm{T} / \mathrm{mv}$ & $\mathrm{T} / \mathrm{mv}$ \\
\hline \multirow{2}{*}{ 1 week } & Normal control group & 10 & $0.038 \pm 0.038$ & $0.227 \pm 0.101$ \\
& Sham operation group & 10 & $0.052 \pm 0.035$ & $0.252 \pm 0.076$ \\
& Model group & 11 & $0.140 \pm 0.121^{* *}$ & $0.312 \pm 0.151$ \\
\multirow{2}{*}{2 weeks } & Normal control group & 10 & $0.042 \pm 0.035$ & $0.242 \pm 0.102$ \\
& Sham operation group & 10 & $0.042 \pm 0.030$ & $0.232 \pm 0.061$ \\
& Model group & 8 & $0.104 \pm 0.044^{*}$ & $0.234 \pm 0.076$ \\
& Normal control group & 10 & $-0.005 \pm 0.039$ & $0.270 \pm 0.146$ \\
& Sham operation group & 10 & $0.021 \pm 0.051$ & $0.254 \pm 0.082$ \\
& Model group & 9 & $0.088 \pm 0.084$ & $0.242 \pm 0.097$ \\
\hline
\end{tabular}

Note: Compared with sham operation group, ${ }^{*} P<0.05,{ }^{* *} P<0.01$.
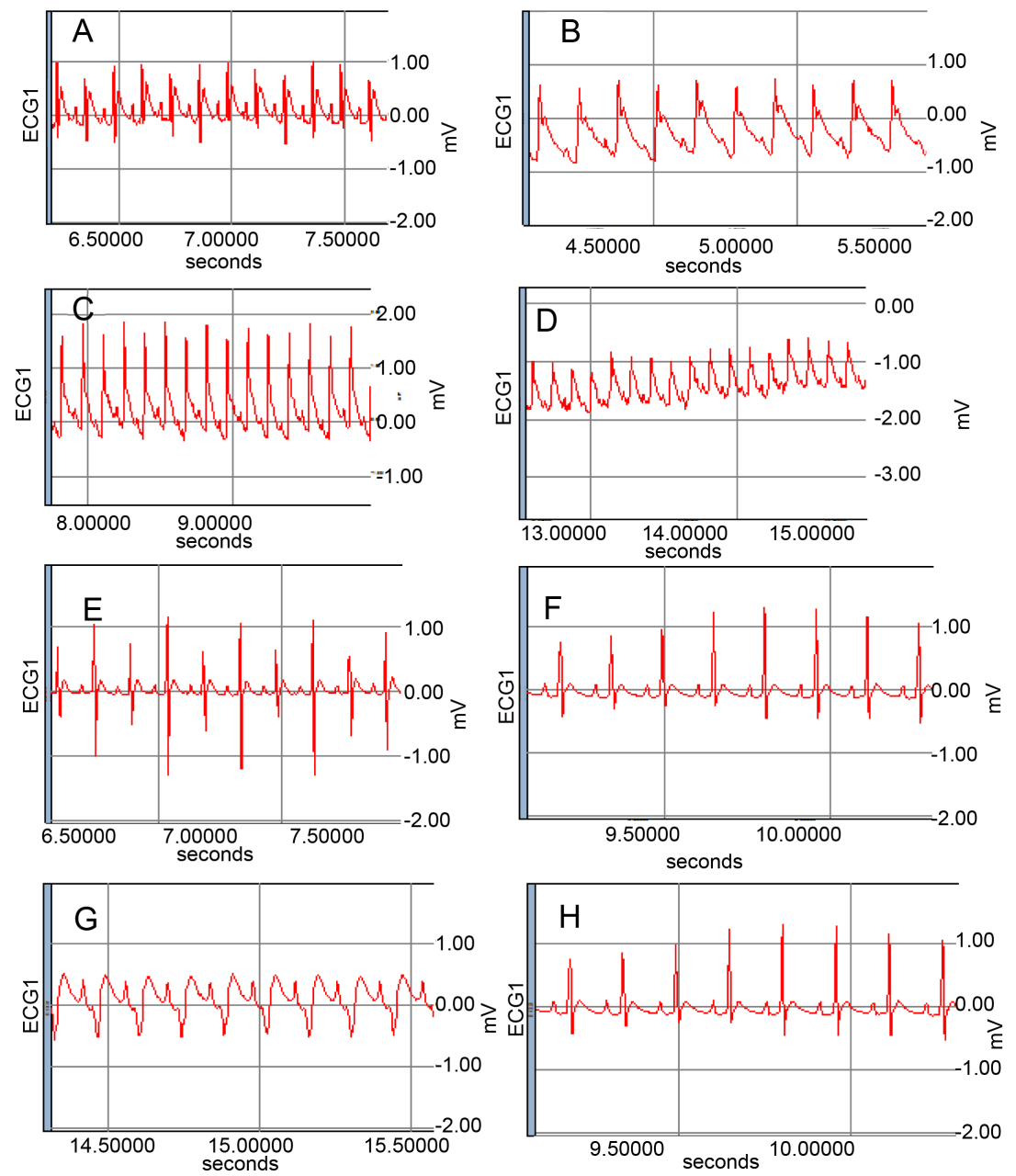

Figure 1. The representive ECG of myocardial ischemia rats induced by coronary artery ligation at different modeling time point. (Note: (A) normal ECG before modeling; (B)-(D) the ECG of typical lesions after modeling; (E) 1 week of control group; (F) 1week of sham operation group; (G) 1 week of model group; (H) 4 weeks of model group). 


\subsection{Cardiac Function}

At 1 and 2 weeks after coronary artery ligation, the cardiac function of rats in model group was significantly changed compared with that of sham operation group, including the reduction of $+\mathrm{dp} / \mathrm{dtmax},-\mathrm{dp} / \mathrm{dtmin}$, LVSP $(P<0.01, P<0.05)$ and the elevation of LVEDP $(P<0.001, P<0.01) .4$ weeks after coronary artery ligation, the cardiac function of rats in model group was significantly changed compared with that of sham operation group, including the reduction of LVSP $(P<0.05)$ and the elevation of LVEDP $(P<0.001)$, the values of -dp/ $\mathrm{dtmin}$ and $+\mathrm{dp} / \mathrm{dtmin}$ were also decreased, but no significant difference was found. The results are shown in Table 3.

\subsection{Myocardial Enzyme Spectrum}

Compared with sham operation group, the myocardial enzyme spectrum in the model group was not significantly different 1 and 2 weeks afer modeling. 4 weeks after coronary ligation, the serum contents of CK-MB, CK, AST and LDH in the model group were significantly higher than those in sham operation group $(P<0.01, P<$ 0.05). The results are shown in Table 4.

\subsection{Organ Index and Myocardial Infarct Size}

Compared with sham operation group, the cardiac and left ventricular indexes of model group were significantly increased at 1 week after modeling $(P<0.001)$, and this increase was lasted until 4 weeks after modeling $(P<$ 0.001). The myocardial infarct size of model group was $30.8 \%$ at 1 week after modeling, and 2 weeks after modeling the myocardial infarct size was about 36\%. The results are shown in Table 5.

\subsection{Pathological Examination}

Normal control group: 1, 2 and 4 weeks after modeling, the epicardium and endocardium of left ventricular were intact, and no thickening, adhesion and inflammatory cell infiltration were found; the arrangement myocardial cells was neat, and no degeneration, necrosis and inflammatory cell infiltration were found, the structure of left ventricular was clear.

Sham operation group: 1, 2 and 4 weeks after modeling, although subepicardial myocardium of individual animal showed focal necrosis and degeneration, there was no significant histopathological difference in sham operation group compared with normal control group.

Table 3. The cardiac function of myocardial ischemia rats induced by coronary artery ligation at different modeling time $(\bar{x} \pm \mathrm{sd})$.

\begin{tabular}{|c|c|c|c|c|c|c|c|}
\hline $\begin{array}{l}\text { Modeling } \\
\text { time }\end{array}$ & Groups & $\mathrm{n}$ & $+\mathrm{dp} / \mathrm{dtmax}$ & -dp/dtmin & LVSP & LVEDP & HR \\
\hline \multirow[t]{3}{*}{1 week } & $\begin{array}{c}\text { Normal control } \\
\text { group }\end{array}$ & 10 & $3713.2 \pm 701.9$ & $-3333.4 \pm 731.2$ & $107.5 \pm 14.7$ & $4.7 \pm 1.4$ & $416.4 \pm 34.8$ \\
\hline & $\begin{array}{c}\text { Sham operation } \\
\text { group }\end{array}$ & 10 & $3765.8 \pm 519.2$ & $-3310.3 \pm 655.7$ & $111.5 \pm 10.6$ & $4.9 \pm 1.9$ & $425.3 \pm 49.9$ \\
\hline & Model group & 11 & $2281.2 \pm 666.9^{* *}$ & $-2238.0 \pm 713.6^{*}$ & $92.0 \pm 14.9^{*}$ & $15.2 \pm 6.0^{* * *}$ & $416.9 \pm 38.8$ \\
\hline \multirow[t]{3}{*}{2 weeks } & $\begin{array}{c}\text { Normal control } \\
\text { group }\end{array}$ & 10 & $3576.7 \pm 615.8$ & $-3186.0 \pm 639.8$ & $129.9 \pm 12.1$ & $4.3 \pm 1.5$ & $415.4 \pm 13.1$ \\
\hline & $\begin{array}{c}\text { Sham operation } \\
\text { group }\end{array}$ & 10 & $3650.1 \pm 713.4$ & $-3481.8 \pm 572.6$ & $125.4 \pm 13.7$ & $4.1 \pm 2.6$ & $422.9 \pm 13.9$ \\
\hline & Model group & 11 & $2321.1 \pm 839.1^{*}$ & $-2547.8 \pm 642.9^{*}$ & $102.1 \pm 12.4^{*}$ & $14.6 \pm 2.2^{* *}$ & $426.4 \pm 22.0$ \\
\hline \multirow[t]{3}{*}{4 weeks } & $\begin{array}{c}\text { Normal control } \\
\text { group }\end{array}$ & 10 & $3562.9 \pm 1698.2$ & $-3391.1 \pm 1322.4$ & $130.5 \pm 12.2$ & $8.9 \pm 5.9$ & $334.8 \pm 51.5$ \\
\hline & $\begin{array}{c}\text { Sham operation } \\
\text { group }\end{array}$ & 10 & $3902.4 \pm 1663.0$ & $-2772.3 \pm 1014.7$ & $129.7 \pm 16.5$ & $5.9 \pm 4.7$ & $348.6 \pm 39.2$ \\
\hline & Model group & 11 & $2202.4 \pm 1017.1$ & $-1757.8 \pm 807.5$ & $106.7 \pm 20.5^{*}$ & $26.7 \pm 9.7^{* * *}$ & $321.1 \pm 71.4$ \\
\hline
\end{tabular}

Note: Compared with sham operation group, ${ }^{*} P<0.05, * * P<0.01,{ }^{* * *} P<0.001$. 
Table 4. The contents of LDH, CK-MB, AST and CK in serum of myocardial ischemia rats induced by coronary artery ligation at different modeling time ( $\bar{x} \pm \mathrm{sd})$.

\begin{tabular}{ccccccc}
\hline Modeling time & Groups & $\mathrm{n}$ & CK-MB & AST & CK & LDH \\
\hline \multirow{2}{*}{1 week } & Normal control group & 10 & $651 \pm 182$ & $165 \pm 25$ & $677 \pm 175$ & $1555 \pm 397$ \\
& Sham operation group & 10 & $587 \pm 128$ & $174 \pm 24$ & $659 \pm 112$ & $1541 \pm 360$ \\
& Model group & 11 & $672 \pm 106$ & $177 \pm 18$ & $698 \pm 109$ & $1707 \pm 287$ \\
& Normal control group & 10 & $785 \pm 171$ & $164 \pm 31$ & $715 \pm 213$ & $1274 \pm 536$ \\
& Sham operation group & 10 & $682 \pm 271$ & $163 \pm 50$ & $729 \pm 499$ & $1137 \pm 698$ \\
& Model group & 11 & $737 \pm 319$ & $148 \pm 22$ & $711 \pm 159$ & $1279 \pm 539$ \\
& Normal control group & 10 & $428 \pm 102$ & $93 \pm 29$ & $215 \pm 44$ & $368 \pm 155$ \\
& Sham operation group & 10 & $379 \pm 128$ & $94 \pm 11$ & $204 \pm 59$ & $321 \pm 273$ \\
& Model group & 11 & $907 \pm 500^{* *}$ & $119 \pm 30^{* *}$ & $421 \pm 223^{* *}$ & $1102 \pm 597^{* *}$ \\
\hline
\end{tabular}

Note: Compared with sham operation group, ${ }^{*} P<0.05,{ }^{* *} P<0.01$.

Table 5. The organ index and myocardial infarct size of myocardial ischemia rats induced by coronary artery ligation at different modeling time ( $\bar{x} \pm \mathrm{sd})$.

\begin{tabular}{|c|c|c|c|c|c|c|c|}
\hline $\begin{array}{l}\text { Modeling } \\
\text { time }\end{array}$ & Groups & $\begin{array}{l}\text { Dose/(crude drug } \\
\text { g/kg) }\end{array}$ & $\mathrm{n}$ & Heart index/(g/g \%) & $\begin{array}{c}\text { Left ventricular } \\
\text { index/(g/g \%) }\end{array}$ & $\mathrm{n}$ & $\begin{array}{c}\text { Myocardial } \\
\text { infarct size/\% }\end{array}$ \\
\hline \multirow[t]{3}{*}{1 week } & $\begin{array}{c}\text { Normal control } \\
\text { group }\end{array}$ & - & 10 & $0.361 \pm 0.034$ & $0.256 \pm 0.037$ & 5 & 0 \\
\hline & $\begin{array}{c}\text { Sham operation } \\
\text { group }\end{array}$ & - & 10 & $0.357 \pm 0.037$ & $0.252 \pm 0.027$ & 5 & 0 \\
\hline & Model group & 10.4 & 11 & $0.437 \pm 0.042^{* * *}$ & $0.299 \pm 0.033^{* * *}$ & 6 & $30.8 \pm 8.6$ \\
\hline \multirow[t]{3}{*}{2 weeks } & $\begin{array}{l}\text { Normal control } \\
\text { group }\end{array}$ & - & 10 & $0.365 \pm 0.036$ & $0.235 \pm 0.030$ & 5 & 0 \\
\hline & $\begin{array}{c}\text { Sham operation } \\
\text { group }\end{array}$ & - & 10 & $0.358 \pm 0.017$ & $0.230 \pm 0.029$ & 5 & 0 \\
\hline & Model group & 10.4 & 11 & $0.413 \pm 0.034^{* * *}$ & $0.290 \pm 0.017^{* * *}$ & 6 & $36.5 \pm 13.0$ \\
\hline \multirow[t]{3}{*}{4 weeks } & $\begin{array}{l}\text { Normal control } \\
\text { group }\end{array}$ & - & 10 & $0.331 \pm 0.019$ & $0.252 \pm 0.017$ & 5 & 0 \\
\hline & $\begin{array}{c}\text { Sham operation } \\
\text { group }\end{array}$ & - & 10 & $0.331 \pm 0.037$ & $0.253 \pm 0.029$ & 5 & 0 \\
\hline & Model group & 10.4 & 11 & $0.426 \pm 0.053^{* * *}$ & $0.317 \pm 0.030^{* * *}$ & 6 & $36.4 \pm 8.7$ \\
\hline
\end{tabular}

Note: Compared with sham operation group, ${ }^{* * * *} P<0.001$.

Model group: 1, 2 and 4 weeks after modeling, the myocardial lesions of model group were significantly different from those of the normal control group and sham operation group $(P<0.01)$.

1 week after ligation, in individual animal (1/5), small myocardial cells under epicardium were swelling, degenerative and necrotic, a small amount of inflammatory cells were infiltrated; in most animals (4/5), patchy myocardial cells were infracted, accompanied by hyperplasia of granulation tissue and a small amount of inflammatory cell infiltration, serious myocardial necrosis even formed transmural myocardial infarction, granulation tissue was significantly hyperplastic and ventricular wall was significantly thin.

2 weeks after ligation, in some animals (2/5), small patchy myocardial cells were necrotic, fibrous tissue was mildly hyperplastic, a small amount of inflammatory cell were infiltrated; in the majority of animals (3/5) myocardial lesions were serious, patchy or even large patchy myocardial cells were infracted and ventricular wall was thin.

4 weeks after ligation, in some animals (2/5), small patchy myocardial cells under epicardium were swelling, 
degenerative, necrotic and a small amount of inflammatory cells were infiltrated; in partial animals (3/5), patchy myocardial cells were infracted, accompanied by hyperplasia of fibrous tissue and a small amount of inflammatory cell infiltration.

The results are shown in Figure 2 and Table 6.

\section{Discussion}

Myocardial ischemia induced by ligation of rat coronary artery is the most widely used method, but its use is limited by the high mortality of this method. It has been reported that the survival rate of animals can be improved by controlling the anesthesia and using the non-invasive mouth intubation [12].

Because of the characteristics and merits of ECG in the diagnosis of acute myocardial ischemia and myocardial infarction, ECG is an important method for monitoring myocardial ischemia and myocardial infarction [13] [14]. In a short period after myocardial ischemia induced by left coronary artery ligation, the ST segment of I, II, a VL leads in ECG is significantly higher, and the amplitude of T wave is also increased. Our results showed that there were significant differences in the value of ST segment elevation after 1 and 2 weeks of modeling, but the increase of ST segment was not significantly different from the sham operation group 4 weeks after modeling. Some researchers reported ST-T value in ECG 5, 10, 15 and 20 days after ligation was approximately same, including the value of ST-T was recovered to preoperative level, or ST segment shifted down, and the amplitude of $\mathrm{T}$ wave was decreased or inverted [6]. In our experiment, the ECG of myocardial infarction rats was also slowly recovered.

1 week after ligation of left coronary artery, compared with sham operation group, the indicators of the model group were significantly changed, including the decrease of $+\mathrm{dp} / \mathrm{dtmax},-\mathrm{dp} / \mathrm{dtmin}, \mathrm{LVSP}$ and the increase of LVEDP, suggesting that myocardial systolic function and diastolic function were decreased 1 week after modeling, return blood volume was increased, left ventricular blood outflow tract was blocked, and heart preload and afterload were increased [15]. Two weeks after modeling, due to the self-compensation of rats, the cardiac function showed self-recovery trend, but the indexes of heart function still showed significant difference. Four weeks after modeling, the self-recovery was further increased, compared with sham operation group, in addition to LVSP and LVEDP, the rest indexes had no statistically significant difference.

The CK, AST, CK-MB and LDH are biological enzymes in cells. CK-MB whose distribution has a certain tissue specificity is mainly distributed in myocardium. Myocardial ischemia and hypoxia induced by coronary artery occlusion lead to myocardial cell injury and cell membrane integrity destruction, so the enzymes are released into the blood. The activity of the enzyme in serum is significantly increased, and the degree of elevation is proportional to the degree of myocardial damage [16]. Our results showed that compared with sham operation group, 1 - 2 weeks after modeling, there was no significant difference in CK, CK-MB, LDH, AST levels in the

Table 6. The degree of myocardial lesion of myocardial ischemia rats induced by coronary artery ligation at different modeling time point.

\begin{tabular}{|c|c|c|c|c|c|c|c|c|}
\hline \multirow{2}{*}{ Modeling time } & \multirow{2}{*}{ Groups } & \multirow{2}{*}{$\mathrm{n}$} & \multicolumn{5}{|c|}{ Degree of myocardial lesion } & \multirow{2}{*}{ Rank } \\
\hline & & & - & + & ++ & +++ & ++++ & \\
\hline \multirow[t]{3}{*}{1 week } & Normal control group & 5 & 5 & 0 & 0 & 0 & 0 & 5.5 \\
\hline & Sham operation group & 5 & 5 & 0 & 0 & 0 & 0 & 5.5 \\
\hline & Model group & 5 & 0 & 1 & 0 & 2 & 2 & $13^{* *}$ \\
\hline \multirow[t]{3}{*}{2 weeks } & Normal control group & 5 & 5 & 0 & 0 & 0 & 0 & 5 \\
\hline & Sham operation group & 5 & 4 & 1 & 0 & 0 & 0 & 6 \\
\hline & Model group & 5 & 0 & 0 & 2 & 2 & 1 & $13^{* *}$ \\
\hline \multirow[t]{3}{*}{4 weeks } & Normal control group & 5 & 5 & 0 & 0 & 0 & 0 & 5 \\
\hline & Sham operation group & 5 & 4 & 1 & 0 & 0 & 0 & 6 \\
\hline & Model group & 5 & 0 & 0 & 2 & 3 & 0 & $13^{* *}$ \\
\hline
\end{tabular}

Note: Compared with sham operation group, ${ }^{* * *} P<0.01$. 


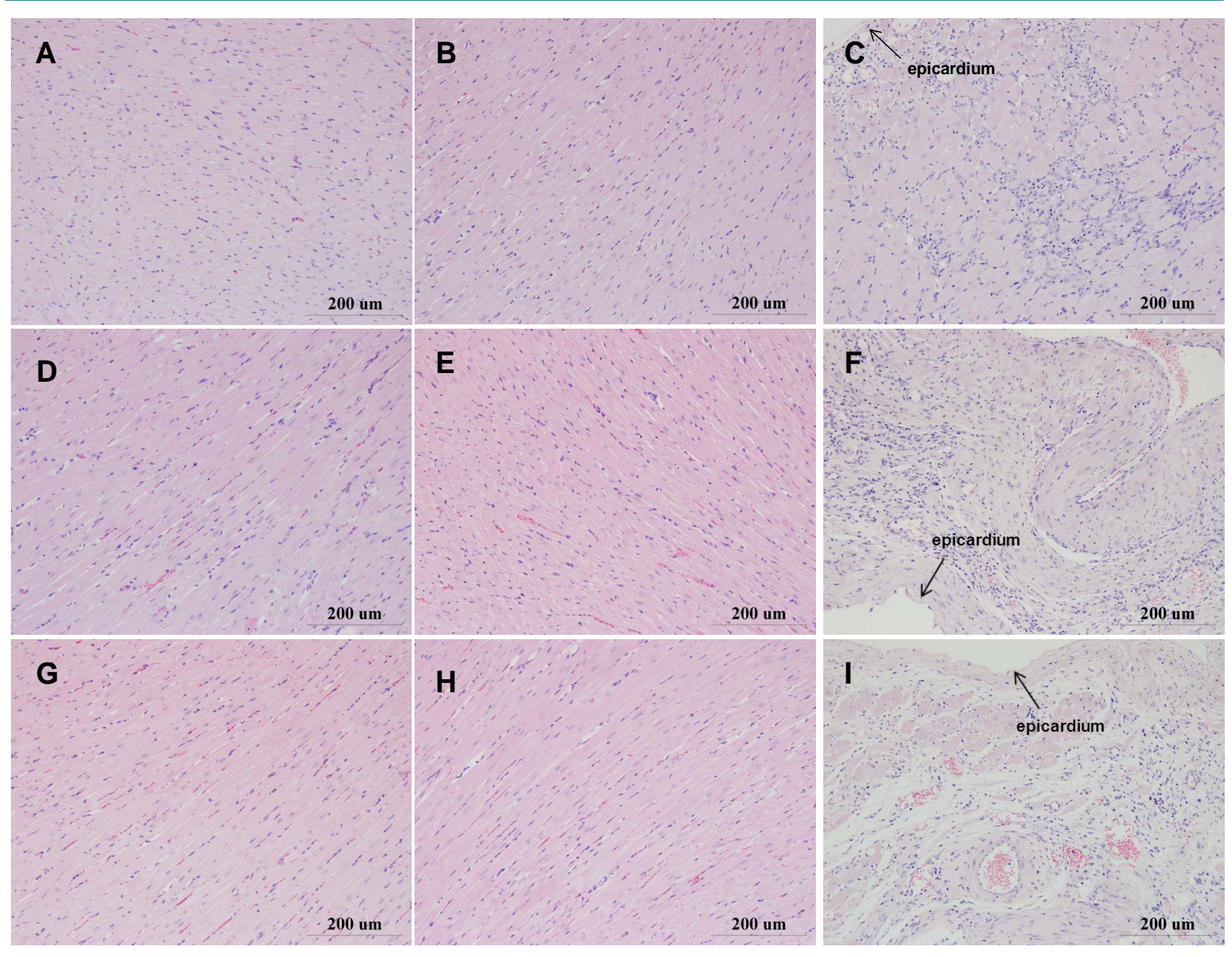

Figure 2. The representive pathological pictures of myocardial ischemia rats induced by coronary artery ligation at different modeling time point. Each photo was taken from left ventricular wall near the epicardium (Note: (A)-(C): respectively represent the pathological picture of control group, sham operation group and model group 1 week after modeling; (D)-(F): respectively represent the pathological picture of control group, sham operation group and model group after 2 weeks of modeling; (G)-(I): respectively represent the pathological picture of control group, sham operation group and model group after 4 weeks of modeling; H\&E $\times 200$ ).

model group; 4 weeks after modeling, CK-MB, CK, AST, LDH was significantly increased. From 1 to 4 weeks, the myocardial enzyme spectrum showed an increasing trend. It maybe myocardial enzymes, including CK, AST, LDH, CK-MB, can show statistically significant differences until the model group animals form a relatively constant myocardial infarction.

Heart index, left ventricular index, myocardial infarction area and pathological results suggest that after 1 week of modeling, the left ventricular index of model group was increased, the infarct size was around $30 \%$, there were a lot of myocardium cells necrosis and granulation tissue hyperplasia in infarct area; with the modeling time extended, 2 to 4 weeks after modeling, the left ventricular and heart indexes of model group were significantly increased, forming a constant infarct size, the left ventricular wall was significantly thin, and fibrous tissue in the infarct area was obvious under the light microscope.

The results of this experiment showed that the changes of ECG, cardiac function and myocardial pathological changes were the most obvious 1 week after modeling, at the 4th week, the ECG and heart function of the model animals had been recovered, but the range of myocardial infarction was relatively constant, and the organ index and myocardial enzyme spectrum were changed obviously. The results of this study suggest that in the evaluation of drug efficacy, we can choose different time points to observe anti-myocardial ischemia effect of the subjects from different aspects, so as to better evaluate the therapeutic effect of drugs. 


\section{Conclusion}

In summary, we conclude the indexes of myocardial ischemia induced by coronary artery ligation in rats. They are different at different time points. The ECG and heart function indexes of rats model have significant change after 1 week, while the myocardial enzymes are significantly increased after 4 weeks, the infarct size, the pathological findings, the left ventricular and heart indexes are significantly changed from 1 to 4 weeks.

\section{Fund Project}

The thesis study belongs to the subject of National Nature Science Foundation of China (90709043).

\section{References}

[1] Su, Y., Wang, L., Zhang, M.Z., et al. (2012) Advances in the Epidemiology of Acute Myocardial Infarction. Chinese Journal of Integrative Medicine on Cardio-/Cerebrovascular Disease, 4, 467-469.

[2] Verdouw, P.D., van den Doel, M.A., de Zeeuw, S., et al. (1998) Animal Models in the Study of Myocardial Ischaemia and Ischaemic Syndromes. Cardiovascular Research, 39, 121-135. http://dx.doi.org/10.1016/S0008-6363(98)00069-8

[3] Zhou, W.W., Lin, L., Chen, J., et al. (2004) Structure of Ischemic Heart Model in Rats by Coronary Artery Ligation. Acta Laboratorium Animalis Scientia Sinica, 12, 226-230.

[4] Liu, D.N., Ma, Q.Q., Huang, X.L., et al. (2010) Electrocardiogram of Healthy Rats and Rats with Acute Myocardial Ischemia. Journal of Medical Internet Research, 39, 11-14.

[5] Yang, J.Y., Zhang, Y.C., Wang, M.J., et al. (2006) Effect on Myocardial Infarction Size and Cardiac Function in Left Coronary Artery Rats of Different Ligation Time. Journal of Yunyang Medical College, 25, 74-77.

[6] He, T. (2007) Pathological Changes of Myocardial Infarction Model in Rats. Guangxi Medical University, Nanning.

[7] Xu, S.Y., Bian, R.L. and Chen, X. (2001) Pharmacology Experiment Methodology. Cardiovascular System Drug Test Method. 3rd Edition, People’s Health Publishing House, Beijing, 976-1059.

[8] Zhang, R.F. and Wang, J.S. (2004) Some Problems of Establishing Rat Model of Myocardial Infarction. Journal of Shanxi Medical University, 35, 13-15.

[9] Abdalla, S., Makhoul, G., Duong, M., et al. (2013) Hyaluronic Acid-Based Hydrogel Induces Neovascularization and Improves Cardiac Function in a Rat Model of Myocardial Infarction. Interactive Cardiovascular and Thoracic Surgery, 17, 767-772. http://dx.doi.org/10.1093/icvts/ivt277

[10] Lujan, H.L. and Dicarlo, S.E. (2008) Sex Differences to Myocardial Ischemia and Beta-Adrenergic Receptor Blockade in Conscious Rats. American Journal of Physiology-Heart and Circulatory Physiology, 294, H1523-H1529. http://dx.doi.org/10.1152/ajpheart.01241.2007

[11] Zhang, Y.J., Yang, S.H., Li, M.H., et al. (2014) Berberine Attenuates Adverse Left Ventricular Remodeling and Cardiac Dysfunction after Acute Myocardial Infarction in Rats: Role of Autophagy. Clinical and Experimental Pharmacology and Physiology, 41, 995-1002. http://dx.doi.org/10.1111/1440-1681.12309

[12] Liu, T., Cao, C.Y., Hui, L.Q., et al. (2013) Protective Effects of Guanxinshu Capsule on Myocardial Ischemia Induced by Ligating Coronary Artery in Rats. Chinese Journal of Experimental Traditional Medical Formulae, 19, 189-193.

[13] Lu, R. (2001) Research Progress of Acute Myocardial infarction by ECG. Journal of Electrocardiology (China), 20, $115-120$.

[14] Zhou, Y. (2008) Progress of Acute Myocardial Infarction. Journal of Practical Electrocardiology, 17, 44-46.

[15] Xu, S.Y., Bian, R.L. and Chen, X. (2001) Pharmacology Experiment Methodology. Cardiac Insufficiency Experiment. 3rd Edition, People's Health Publishing House, Beijing, 1079-1093.

[16] Ye, H.Y., Hu, Z.P., Zhou, L., et al. (2006) Protective Effect of Daidzein on Myocardial Ischemia Injury in Rats. Journal of the Fourth Military Medical University, 27, 221-223. 